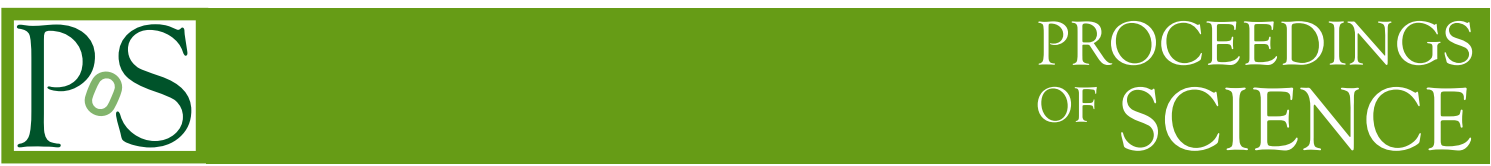

\title{
Spectral densities from the lattice
}

\author{
Paulo J. Silva* \\ Centro de Física Computacional, Departamento de Física, Universidade de Coimbra, 3004-516 \\ Coimbra, Portugal \\ E-mail: psilva@teor.fis.uc.pt
}

\section{David Dudal}

Ghent University, Department of Physics and Astronomy, Krijgslaan 281-S9, 9000 Gent, Belgium

E-mail: david.dudal@ugent.be

\section{Orlando Oliveira}

Centro de Física Computacional, Departamento de Física, Universidade de Coimbra, 3004-516

Coimbra, Portugal

E-mail: orlando@fis.uc.pt

We discuss a method to extract the Källén-Lehmann spectral density of a particle (be it elementary or bound state) propagator by means of $4 \mathrm{~d}$ lattice data. We employ a linear regularization strategy, commonly known as the Tikhonov method with Morozov discrepancy principle. An important virtue over the popular maximum entropy method is the possibility to also probe unphysical spectral densities, as, for example, of a confined gluon. We apply our proposal to the SU(3) glue sector.

31st International Symposium on Lattice Field Theory LATTICE 2013

July 29 - August 3, 2013

Mainz, Germany

\footnotetext{
*Speaker.
} 


\section{Introduction}

In recent years, there has been an renewed interest in the infrared behaviour of the Landau gauge Yang-Mills propagators, related to the gluon confinement phenomenon. In particular, lattice studies of the Landau gauge gluon propagator

$$
D_{\mu \nu}^{a b}(\hat{q})=\delta^{a b}\left(\delta_{\mu v}-\frac{q_{\mu} q_{v}}{q^{2}}\right) D\left(q^{2}\right),
$$

have been performed at large volumes, with the propagators reaching a finite non-zero value in the infrared region. While the simulations have been performed using volumes as large as $(27 \mathrm{fm})^{4}$ for the SU(2) gauge group [1] and $(17 \mathrm{fm})^{4}$ for the SU(3) gauge group [2], the lattice spacing used in the simulations was quite big, being $0.22 \mathrm{fm}$ for $\mathrm{SU}(2)$ and $0.18 \mathrm{fm}$ for $\mathrm{SU}(3)$. In a recent paper [3] by some of us, numerical evidence has been given that a large lattice spacing also changes the propagator in the infrared region.

Gluons are not physical particles. Besides the mere question of the infrared behaviour of their propagator, one would also like to identify signs of gluon confinement in this two-point function.

It is already known, from lattice simulations $[4,5,6,7]$, that the Landau gauge gluon propagator displays a violation of spectral positivity. This implies that the gluon cannot appear as a free asymptotic S-matrix state and may be viewed as an indication of gluon confinement. A mini-review about gluon positivity violation can be found in [8].

Lattice studies of positivity proceed by studying the Schwinger function

$$
C(t)=\int_{-\infty}^{\infty} \frac{\mathrm{d} p}{2 \pi} D\left(p^{2}\right) \exp (-i p t) .
$$

Assuming a Källén-Lehmann spectral representation for the gluon, we get

$$
C(t)=\int_{0}^{\infty} \mathrm{d} \omega \rho\left(\omega^{2}\right) e^{-\omega t},
$$

a quantity shown in Figure 1. Note that, although $C(t)<0$ implies a negative spectral density $\rho\left(\omega^{2}\right)$, and hence positivity violation, a positive $C(t)$ says nothing about the sign of the spectral density.

\section{Gluon spectral densities}

Generally speaking, if $\mathscr{G}\left(p^{2}\right) \equiv\langle\mathscr{O}(p) \mathscr{O}(-p)\rangle$ is an Euclidean momentum-space propagator of a (scalar) physical degree of freedom, then it must have a Källén-Lehmann spectral representation

$$
\mathscr{G}\left(p^{2}\right)=\int_{0}^{\infty} \mathrm{d} \mu \frac{\rho(\mu)}{p^{2}+\mu},
$$

with $\rho(\mu) \geq 0$ for $\mu \geq 0$. The spectral density contains information on the masses of physical states described by the operator $\mathscr{O}$.

Our goal is to compute the spectral density $\rho(\mu)$ from the propagator $\mathscr{G}\left(p^{2}\right)$. We can express eq. (2.1) as a double Laplace transform $\mathscr{G}=\mathscr{L}^{2} \hat{\rho}=\mathscr{L}^{*} \hat{\rho}$ where $(\mathscr{L} f)(t) \equiv \int_{0}^{\infty} \mathrm{d} s e^{-s t} f(s)$. This is a notorious ill-posed problem. Note that for $\mathscr{G}\left(p^{2}\right)$ one usually has a set of data points with error bars. 


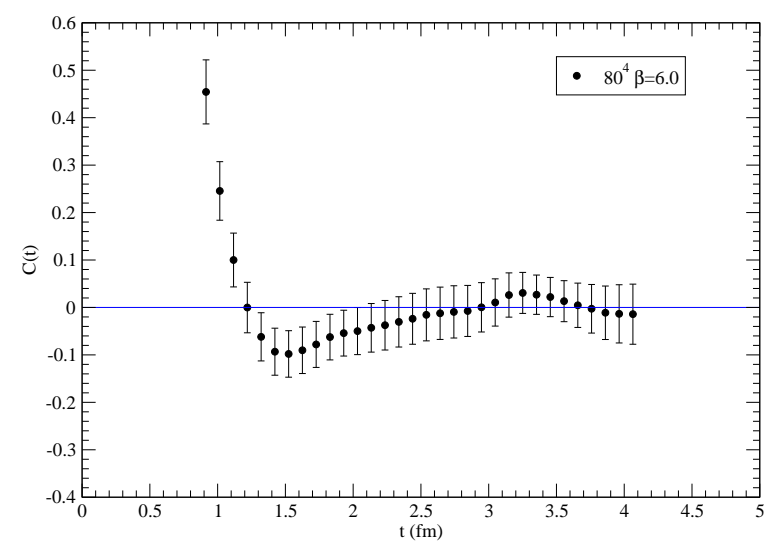

Figure 1: Temporal correlator for the gluon propagator computed using $80^{4} \beta=6.0$ lattice data.

A popular approach to compute spectral functions has been the maximum entropy method [9]. Here we use an alternative approach, based on Tikhonov regularization with Morozov discrepancy principle. For details see our previous papers [10, 11, 12].

To compute the spectral density we consider the integral equation

$$
\int_{0}^{\infty} \mathrm{d} t \rho(t) \frac{\ln \frac{z}{t}}{z-t}+\lambda \rho(z)=\int_{0}^{\infty} \mathrm{d} t \frac{\mathscr{D}(t)}{t+z}
$$

where for $\mathscr{D}(t)$ we use lattice data in momentum space for the gluon propagator computed in a $80^{4}$ volume, with $\beta=6.0$ [3]. In a loose way of speaking, we search for a solution to the integral eq. (2.1) that is sufficiently close in norm to the exact but unknown spectral function. This "sufficiently close" translates into a variational problem, which solution fulfills the foregoing eq. (2.2). The rôle of the parameter $\lambda>0$ is to regularize the ill-posed nature of the problem. For practical purposes, the finite set of data points was interpolated using splines. Furthermore, one has to impose IR and UV cut-offs, although we consider a 1-loop perturbative behaviour after $p_{\text {max }}^{\text {(latt) }}$. The integrals in eq. (2.2) are computed using Gauss-Legendre quadrature, and the discretization of eq. (2.2) leads to a linear system one has to solve in order to compute the spectral density.

In Figures 2, 3, and 4 we study how the solution to eq. (2.2) depends on the number of GaussLegendre (GL) points, and on the IR and UV cut-offs. In what concerns the number of GL points and the UV cut-off, the results for the spectral density (left-hand plots) show that they do not have a big influence in the results. However, we see some differences in the results if we do not consider a UV tail beyond the maximum momentum available in the lattice simulation considered here. Nevertheless, the reconstruction of the input propagator (right-hand plots) apparently does not distinguish the several spectral functions.

On the other hand, a change in the IR cut-off has a dramatic effect in the computed spectral function. Note that in Figure 4, the 'S' values refer to the scale factors needed to fit the spectral density in the interval $[-1,1]$. Despite this, the reconstructed propagators are still indistinguishable.

This study leaded us to try to find out a clever way to compute the spectral density, and hopefully an updated method arose - see [12] for details. Although there are natural differences in the 


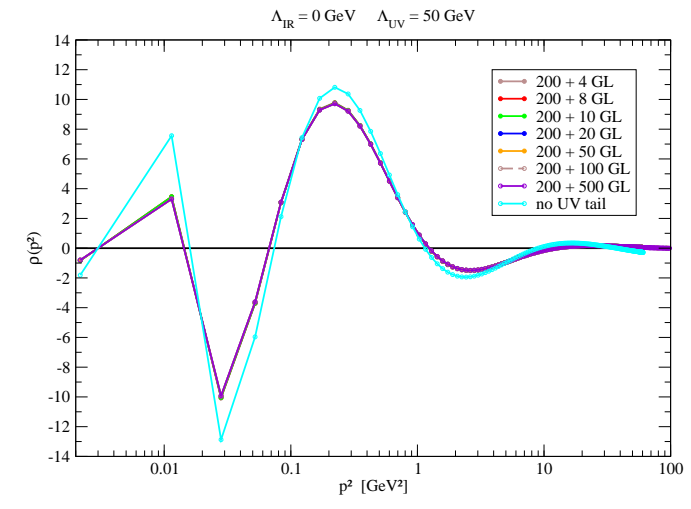

(a) Spectral density.

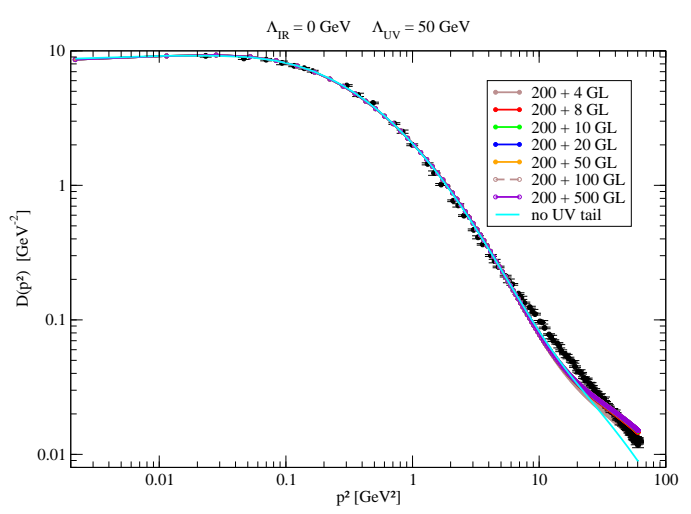

(b) Reconstructed propagator.

Figure 2: Results for the spectral density and for the reconstructed propagator with a different number of Gauss-Legendre points.

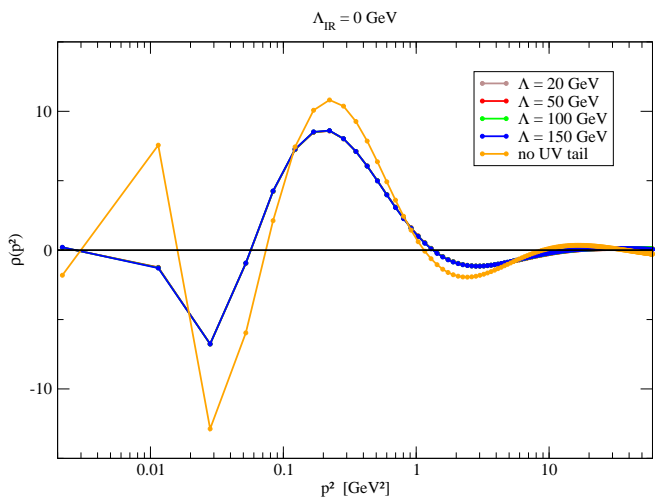

(a) Spectral density.

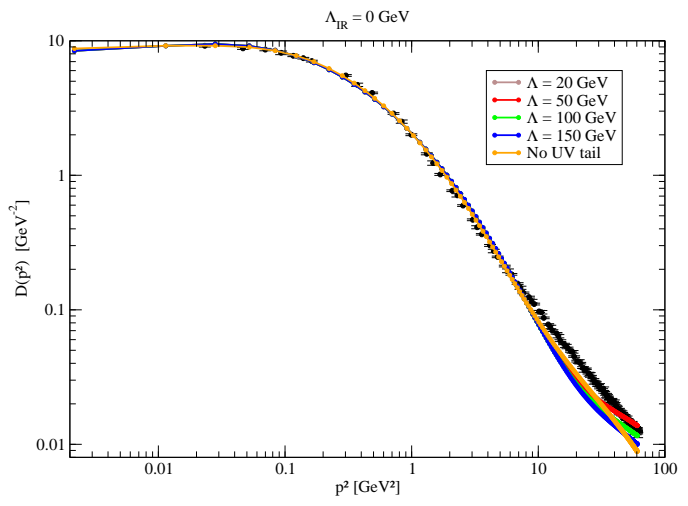

(b) Reconstructed propagator.

Figure 3: The effect of changing the UV cutoff.

deep infrared region due to the different choices of the IR cut-off $\mu_{0}$, the results are much more stable if compared with previous results - see Figure 5. Moreover, the IR cut-off is now interrelated with the Tikhonov parameter $\lambda$ - see [12] for details.

Given recent results for the gluon spectral function obtained from the solution of the DysonSchwinger equations in the complex momentum plane [14], a comparison with our results is in order. It turns out that there are fundamental differences between the results of the two works. In particular, we do not see evidence for the sharp peak reported in [14]. Furthermore, our results show a violation of positivity setting in for small momenta, whereas in [14] positivity violation occurs only for momenta above $600 \mathrm{MeV}$.

\section{Results at finite temperature}

In this section, we consider lattice results for the gluon propagator at finite temperature, and study positivity violation through the computation of the Schwinger and spectral functions.

The lattice setup for the simulations at finite temperature considered here is reported in [13]. 


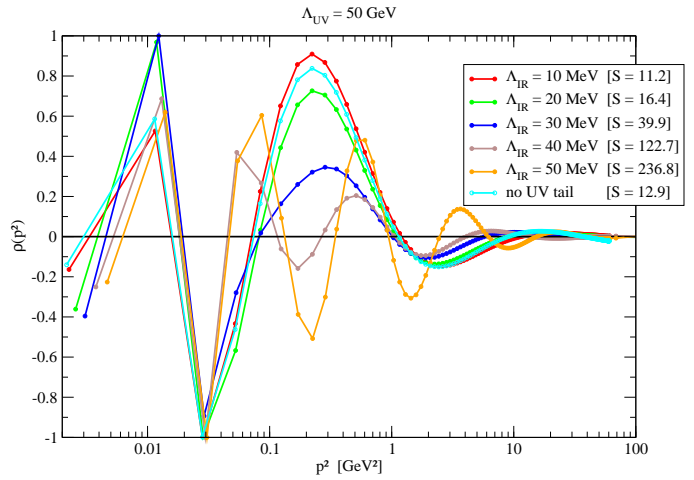

(a) Spectral density.

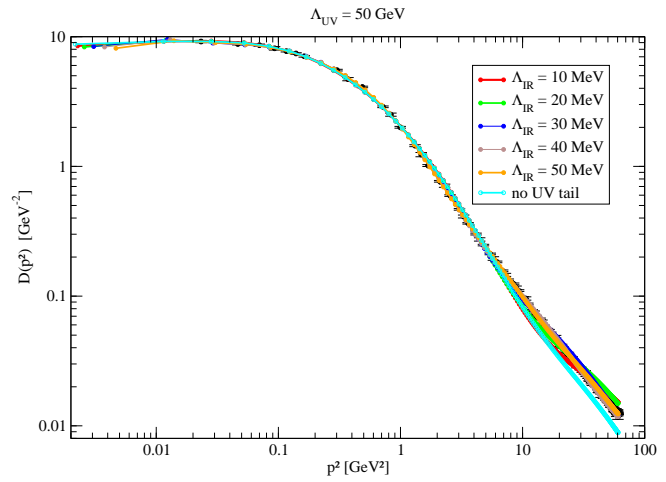

(b) Reconstructed propagator.

Figure 4: The effect of changing the IR cutoff.

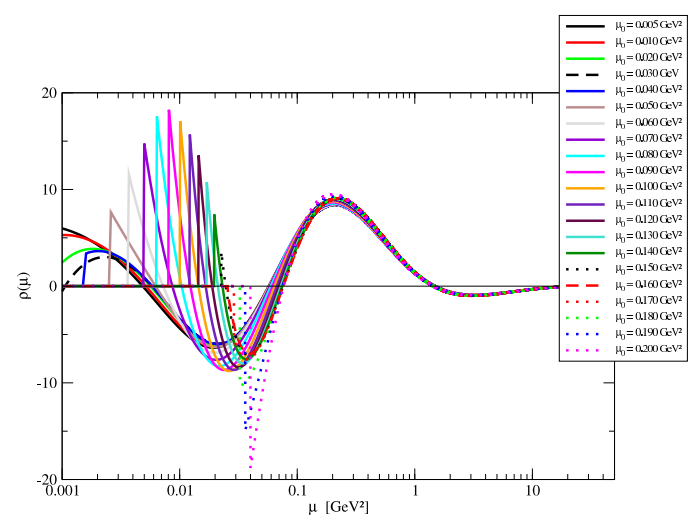

(a) Spectral density.

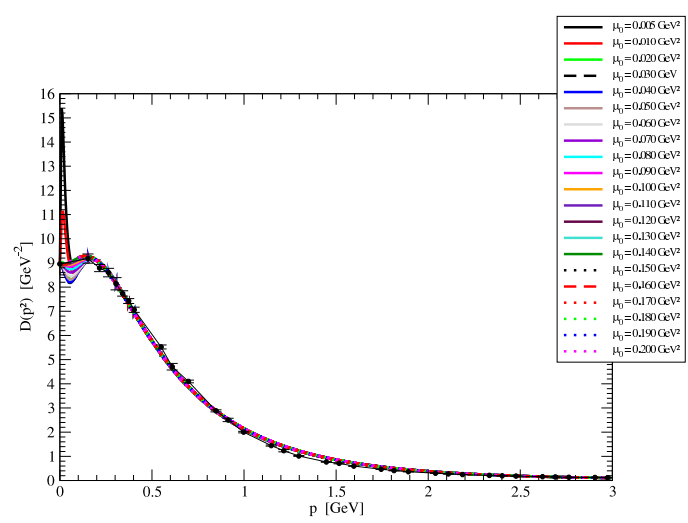

(b) Reconstructed propagator.

Figure 5: Results obtained using the updated method presented in [12].

\subsection{Positivity violation}

In this subsection, the temporal correlator defined in eq. (1.2) is computed for the longitudinal and transverse components of the gluon propagator for all available temperatures. From the results shown in Figures 6 and 7, it turns out that positivity is violated for both transverse and longitudinal components at all temperatures. Note that, in what concerns the transverse propagator, the time scale for positivity violation decreases with the temperature - see Figure 8. This suggests that, for sufficiently high temperatures, transverse gluons can behave as quasi-particles.

\subsection{Spectral densities}

In this subsection, we consider the spectral densities, computed from eq. (2.2), for the longitudinal component - see Figure 9. Notice that the energy scale at which the axis is crossed, increases with temperature. This means, again, that for sufficiently high temperatures, longitudinal gluons may be considered quasi-particles. 

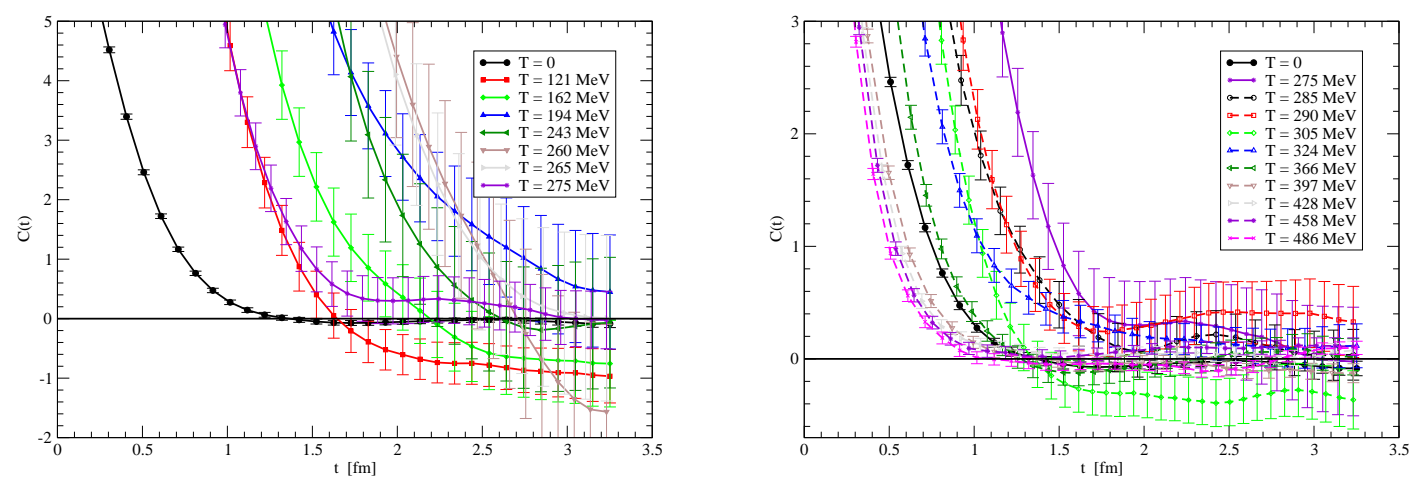

Figure 6: Temporal correlator for the longitudinal component.
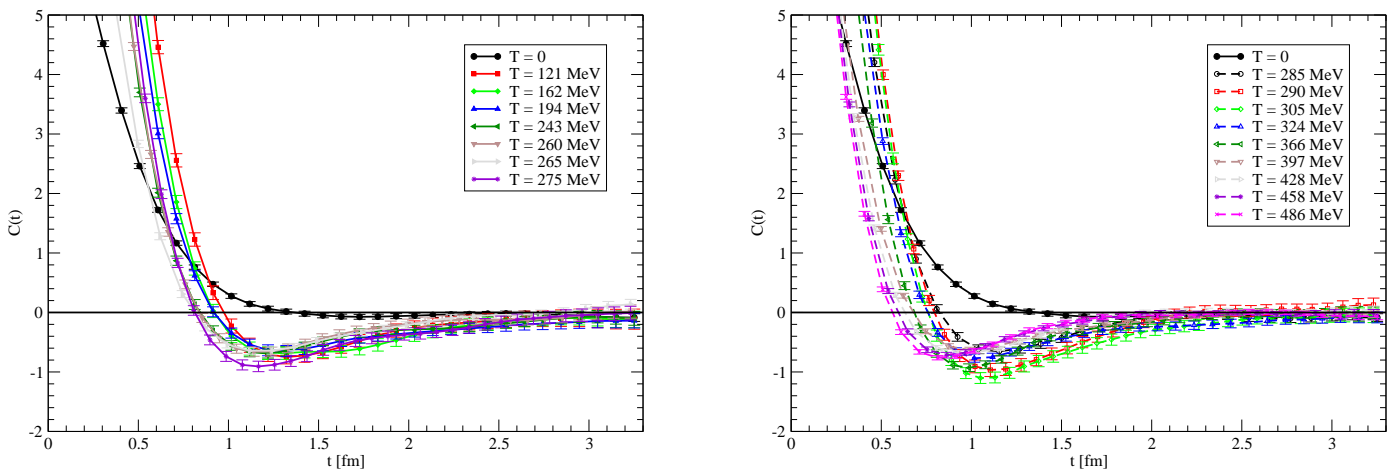

Figure 7: Temporal correlator for the transverse component.

\section{Acknowledgments}

O. Oliveira and P. J. Silva acknowledge support by FCT via projects CERN/FP/123612/2011, CERN/FP/123620/2011, and PTDC/FIS/100968/2008, developed under the initiative QREN financed by the UE/FEDER through the Programme COMPETE - Programa Operacional Factores de Competitividade. P. J. Silva is also supported by FCT grant SFRH/BPD/40998/2007. D. Dudal acknowledges financial support from the Research-Foundation Flanders (FWO Vlaanderen) via the Odysseus grant of F. Verstraete.

\section{References}

[1] A. Cucchieri and T. Mendes, PoS (LATtiCE 2007) 297.

[2] I. L. Bogolubsky, E. M. Ilgenfritz, M. Muller-Preussker and A. Sternbeck, Phys. Lett. B 676 (2009) 69.

[3] O. Oliveira and P. J. Silva, Phys. Rev. D 86 (2012) 114513.

[4] A. Cucchieri, T. Mendes and A. R. Taurines, Phys. Rev. D 71 (2005) 051902.

[5] C. Aubin and M. C. Ogilvie, Phys. Rev. D 70 (2004) 074514. 


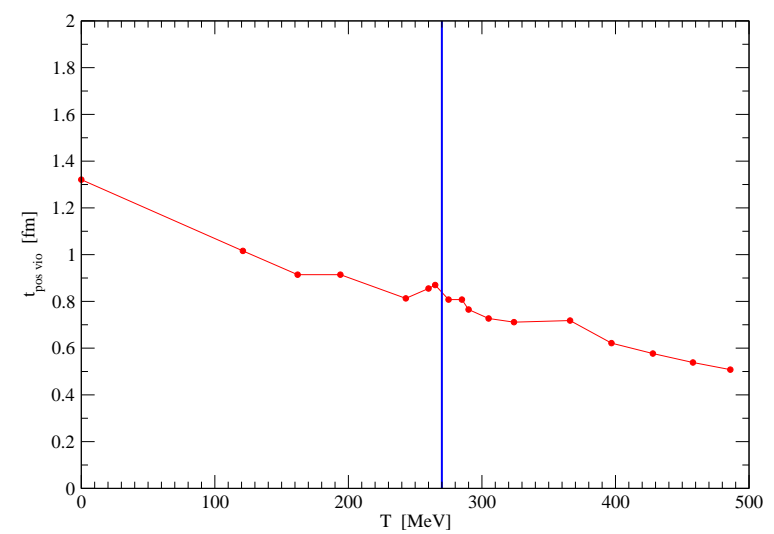

Figure 8: Positivity violation scale for the transverse propagator.
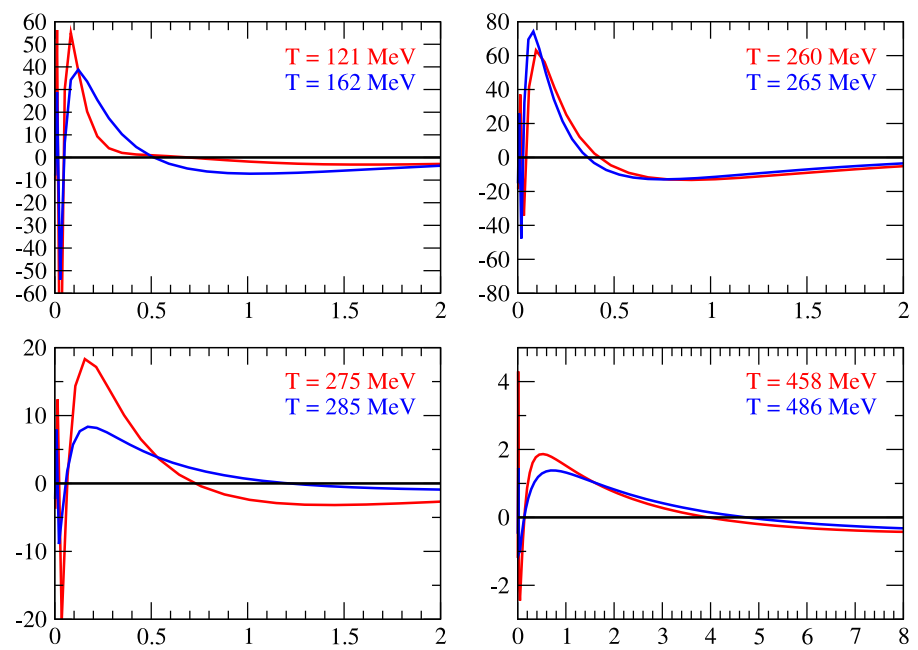

Figure 9: Longitudinal propagator spectral densities.

[6] P. J. Silva and O. Oliveira, PoS (LAT2006) 075.

[7] P. O. Bowman, U. M. Heller, D. B. Leinweber, M. B. Parappilly, A. Sternbeck, L. von Smekal, A. G. Williams and J.-b. Zhang, Phys. Rev. D 76 (2007) 094505.

[8] J. M. Cornwall, arXiv:1310.7897 [hep-ph].

[9] M. Asakawa, T. Hatsuda and Y. Nakahara, Prog. Part. Nucl. Phys. 46 (2001) 459.

[10] O. Oliveira, D. Dudal and P. J. Silva, PoS (Lattice 2012) 214.

[11] D. Dudal, P. J. Silva and O. Oliveira, PoS (Confinement X) 033.

[12] D. Dudal, O. Oliveira and P. J. Silva, arXiv:1310.4069 [hep-lat].

[13] P. J. Silva, O. Oliveira, P. Bicudo and N. Cardoso, arXiv:1310.5629 [hep-lat].

[14] S. Strauss, C. S. Fischer and C. Kellermann, Phys. Rev. Lett. 109 (2012) 252001. 